\title{
New Signals of Quark-Gluon-Hadron Mixed Phase Formation
}

\author{
K.A. Bugaev ${ }^{1, *}$, V.V. Sagun ${ }^{1}$, A.I. Ivanytskyi ${ }^{1}$, D.R. Olinychenko ${ }^{1,2}$, E.-M. Ilgenfritz ${ }^{3}$, \\ E.G. Nikonov ${ }^{4}$, A.V. Taranenko ${ }^{5}$ and G.M. Zinovjev ${ }^{1}$ \\ ${ }^{1}$ Bogolyubov Institute for Theoretical Physics of the National Academy of Sciences of Ukraine, \\ Metrologichna str. $14^{B}$, Kiev 03680, Ukraine \\ ${ }^{2}$ FIAS, Goethe University, Ruth-Moufang Str. 1, 60438 Frankfurt upon Main, Germany \\ ${ }^{3}$ Bogolyubov Laboratory of Theoretical Physics, JINR, 141980 Dubna, Russia \\ ${ }^{4}$ Laboratory for Information Technologies, JINR, 141980 Dubna, Russia \\ ${ }^{5}$ National Research Nuclear University "MEPhI" (Moscow Engineering Physics Institute), \\ Kashirskoe Shosse 31, 115409 Moscow, Russia
}

\begin{abstract}
Here we present several remarkable irregularities at chemical freeze-out which are found using an advanced version of the hadron resonance gas model. The most prominent of them are the sharp peak of the trace anomaly existing at chemical freeze-out at the center of mass energy $4.9 \mathrm{GeV}$ and two sets of highly correlated quasi-plateaus in the collision energy dependence of the entropy per baryon, total pion number per baryon, and thermal pion number per baryon which we found at the center of mass energies 3.8-4.9 GeV and 7.6-10 GeV. The low energy set of quasi-plateaus was predicted a long time ago. On the basis of the generalized shock-adiabat model we demonstrate that the low energy correlated quasi-plateaus give evidence for the anomalous thermodynamic properties inside the quarkgluon-hadron mixed phase. It is also shown that the trace anomaly sharp peak at chemical freeze-out corresponds to the trace anomaly peak at the boundary between the mixed phase and quark gluon plasma. We argue that the high energy correlated quasi-plateaus may correspond to a second phase transition and discuss its possible origin and location. Besides we suggest two new observables which may serve as clear signals of these phase transformations.
\end{abstract}

PACS 25.75.Nq Quark deconfinement, quark-gluon plasma production, phase transitions 12.40.Ee Statistical models 


\section{NEW IRREGULARITIES VS NEW SIGNALS OF QGP}

One of the key problems of modern physics of heavy ion collisions is formulation of physically justified signals of the quark-gluon-hadron mixed phase formation [1]. An essential progress in formulating such signals was achieved recently [2, 3]. Among the new irregularities related to deconfinement the most spectacular one is a sudden jump of the pressure $p$ at chemical freeze-out (CFO) in the narrow range of center of mass collision energies $\sqrt{s_{N N}}=4.3-4.9 \mathrm{GeV}[2$, 4]. The observed pressure jump at CFO is so strong, that the effective number of degrees of freedom, $p / T^{4}$, increases by $70 \%$, while the collision energy changes by $15 \%$ or while the CFO temperature $T$ changes by $30 \%$.

The other remarkable irregularity found at CFO is the sharp peak of the trace anomaly $\delta=\frac{\varepsilon-3 p}{T^{4}}$ (here $\varepsilon$ is energy density) at $\sqrt{s_{N N}}=4.9 \mathrm{GeV}$ [3] (see the upper panel of Fig. 1). One could, of course, conservatively say that this peak is produced by only one data point and that its significance should therefore be considered with some caution. However, one can see that the error bars at $\sqrt{s_{N N}}=4.9 \mathrm{GeV}$ and $\sqrt{s_{N N}}=6.3 \mathrm{GeV}$ do not overlap. Moreover, in [3] we found the one to one correspondence between this trace anomaly peak at CFO and the trace anomaly peak along the shock adibat (see the lower panel of Fig. 1) which appears at the collision energy corresponding exactly to the boundary between the quark gluon plasma (QGP) and quark-gluon-hadron mixed phase. We remind that the shock adiabat model reasonably well describes the hydrodynamic and thermodynamic parameters of the initial state formed in the central nucleus-nucleus collisions in the laboratory energy range $1 \mathrm{GeV} \leq E_{l a b} \leq 30 \mathrm{GeV}[5]$. Note also that the exclusive importance of the found trace anomaly peaks becomes clear, if one recalls that the inflection point/maximum of the trace anomaly at low baryonic densities is traditionally used in lattice QCD to determine the pseudo-critical temperature of the cross-over transition [6].

Besides these irregularities the highly correlated quasi-plateaus in the collision energy dependence of the entropy per baryon $s / \rho_{B}$, total pion number per baryon $\rho_{\pi}^{t o t} / \rho_{B}$, and thermal pion number per baryon $\rho_{\pi}^{t h} / \rho_{B}$ at laboratory energies $6.9-11.6 \mathrm{GeV}$ (i.e. $\sqrt{s_{N N}}=$ $3.8-4.9 \mathrm{GeV}$ ) were also found in [2, 3]. As one can see from the Fig. 2 a clear plateau is only seen in the thermal pion number per baryon while other quantities exhibit quasiplateaus. Nevertheless, these quasi-plateaus are important, since their strong correlation with the plateau in $\rho_{\pi}^{t h} / \rho_{B}$ allowed one to determine their common width in the laboratory 
energy [2, 3]. It is necessary to stress that, in contrast to the most of other CFO irregularities discussed in the modern literature as possible signals of QGP formation, these quasi-plateaus were predicted a long time ago [7 9] on a solid ground of relativistic hydrodynamics. As it was shown in [7-9] and was further argued in [2, 3] the generic reason for an appearance of such quasi-plateaus is in an existence of anomalous thermodynamic properties in the quarkgluon-hadron mixed phase. Usually, in pure gaseous or liquid phases the interaction between the constituents at short distances is repulsive and, hence, at high densities the adiabatic compressibility of matter decreases for increasing pressure. Hence the pure phases have the normal thermodynamic properties. In the mixed phase, however, there appears another possibility to compress matter: by converting the less dense phase into the more dense one. As it was found for several equations of state [2, 3, 7, 9] with a first-order phase transition between hadronic gas and QGP, the phase transition region has anomalous thermodynamic properties, i.e. it corresponds to an increase of the compressibility in the mixed phase at higher pressures.

Using the shock adiabat model [7], it was possible to successfully describe the collision energy dependence of the entropy per baryon [2] found at CFO (see the solid curve in Fig. 22. This allowed one to determine the parameters of the QGP equation of state at high baryonic densities [2] directly from CFO data. It was a great surprise to discover that the whole mixed phase which is rather wide in terms of baryonic densities (it ranges from $2 \mathrm{fm}^{-3}$ at hadronic side of mixed phase to about $10 \mathrm{fm}^{-3}$ at QGP side) is located between two neighboring collision energies available in experiments, i.e. inside the interval $\sqrt{s_{N N}}=4.3-4.9 \mathrm{GeV}$. However, such a narrow collision energy range exactly corresponds to the irregularities discussed above and, moreover, it naturally explains the reason of why the mixed phase was not found during more than three decades of thorough searches. Moreover, on the basis of shock adiabat model it was possible to explain [3] the other irregularities discussed above.

In addition to the low energy set of quasi-plateaus a second set of quasi-plateaus was found at laboratory energies $30-45 \mathrm{GeV}\left(\sqrt{s_{N N}}=7.6-9.2 \mathrm{GeV}\right)[2$, 3] as one can see from Fig. 2. Originally, it was difficult to give a unique interpretation of this high energy set of quasiplateaus [3], since the (generalized) shock adiabat model is no longer reliable at these collision energies [5]. Fortunately, entirely new approach [10] based on a thorough comparison of the quality of experimental data description by the models which do not assume the QGP 
existence (hadron models) and which do assume the QGP formation (QGP models) is able to clarify this question. Thus, quite independently, the meta-analysis performed in [10] led to a conclusion that the mixed phase exists at the same collision energy range which for the first time was found in [2, 3], i.e. it exists between $\sqrt{s_{N N}}=4.3 \mathrm{GeV}$ and $\sqrt{s_{N N}}=4.9 \mathrm{GeV}$. This result not only validates the entire framework of shock adiabat model used in [2, 3], but also it independently justifies the jump of the effective number of degrees of freedom $p / T^{4}$ at $\mathrm{CFO}$ and a sharp peak of the trace anomaly $\delta$ at CFO as reliable signals of QGP formation.

Also the meta-analysis of the quality of data description [10] predicts that the most probable collision energy range of the second phase transition is $\sqrt{s_{N N}}=10-13.5 \mathrm{GeV}$. In other words, it independently demonstrates that interpretation of high energy set of correlated quasi-plateaus as an indicator of phase transition makes sense, but meta-analysis shifts this transition to slightly higher collision energies. It is presently not possible to distinguish two possible explanations of this phenomenon. Its first explanation is that with increasing collision energy the initial states of thermally equilibrated matter formed in nucleus-nucleus collisions move from hadron gas into the mixed phase, then from the mixed phase to QGP and then again they return to the same mixed phase, but at higher initial temperature and lower baryonic density. This scenario corresponds to the case, if QCD phase digram has a critical endpoint 10]. An alternative explanation [10] corresponds to QCD phase diagram with the tricritical endpoint. In this case the second phase transition is the second order phase transition of (partial) chiral symmetry restoration or a transition between quarkyonic matter and QGP [11]. It is remarkable that despite the lack of a single interpretation of the second phase transition at $\sqrt{s_{N N}}=10-13.5 \mathrm{GeV}$ there are strong arguments [10] that the (tri)critical endpoint of QCD phase diagram maybe located within this energy range. In what follows we further develop the hypothesis of two phase transitions, give some additional arguments in favor of (tri)critical endpoint location in the collision energy range specified above and discuss two new observables, which will be a good indicators of the discussed phase transformations. 


\section{HRGM WITH MULTICOMPONENT REPULSION}

The uncovering of novel irregularities discussed in preceding section was achieved using the most successful version of the hadron resonance gas model (HRGM) developed during recent years in [4, 12 15]. Existence of local thermal and chemical equilibrium at CFO allows one to employ the HRGM to describe the hadron yields in the grand canonical formulation [16], i.e. using the temperature $T$, the baryonic $\mu_{B}$, the strange $\mu_{s}$ and the isospin third projection $\mu_{I 3}$ chemical potentials. The chemical potential $\mu_{s}$ is fixed by the condition of zero total strange charge. As usual, the deviation of strange charge from the full chemical equilibrium is accounted by the parameter $\gamma_{s}[17]$. It modifies the thermal density of hadron sort $j \varphi_{j}$ according to the rule $\varphi_{j} \rightarrow \gamma_{s}^{S_{j}} \varphi_{j}$, where $S_{j}$ is total number of strange valence quarks and antiquarks in such hadrons. For instance, $\pi, K$ and $\phi$-mesons have, respectively, $S_{\pi}=0, S_{K}=1$ and $S_{\phi}=2$. In contrast to other versions of HRGM known from the literature (see references in $[4,13,16]$ ) the present one accounts for the hard-core repulsion using different hard-core radii for pions, $R_{\pi}$, kaons, $R_{K}, \Lambda$-hyperons $R_{\Lambda}$, other mesons, $R_{m}$, and baryons, $R_{b}$. The best global fit of 111 independent hadronic multiplicities measured in the collision energy range from $\sqrt{s_{N N}}=2.7 \mathrm{GeV}$ to $\sqrt{s_{N N}}=200 \mathrm{GeV}$ was found for $R_{b}$ $=0.355 \mathrm{fm}, R_{m}=0.4 \mathrm{fm}, R_{\pi}=0.1 \mathrm{fm}, R_{K}=0.38 \mathrm{fm}$ and $R_{\Lambda}=0.11 \mathrm{fm}$ with the quality $\chi^{2} / d o f \simeq 0.95[14]$.

Since the main features of the fitting procedure are well documented in papers 4, 13, here we briefly remind the basic elements of HRGM and formulate the new features of present fit. The most important ingredient of the HRGM with multicomponent hard-core repulsion is introduced via the matrix of second virial coefficients $b_{i j}$ which for the hadrons of radii $r_{i}$ and $r_{j}$ reads as $b_{i j}=\frac{2 \pi}{3}\left(r_{i}+r_{j}\right)^{3}$. The hadrons of $i$-th sort are characterized by the spin-isospin degeneracy $g_{i}$, the mass $m_{i}$ and the width $\Gamma_{i}$ which are taken from the particle tables of the thermodynamic code THERMUS [18]. The HRGM equation of state defines the set of partial pressures $p_{i}$ for each hadronic component $\left(p=\sum_{i} p_{i}\right.$ is total pressure)

$$
p_{i}=T \varphi_{i} \exp \left[\frac{\mu_{i}-2 \sum_{j} p_{j} b_{j i}+\sum_{j l} p_{j} b_{j l} p_{l} / p}{T}\right],
$$

where $\mu_{i}=Q_{i}^{B} \mu_{B}+Q_{i}^{I 3} \mu_{I 3}+Q_{i}^{S} \mu_{S}$ is the full chemical potential of $i$-th sort of hadrons expressed via the charges $\left\{Q_{i}^{A}\right\}$ of $i$-th hadron sort and the corresponding chemical potentials 
$\left\{\mu_{A}\right\}$. In the Boltzmann approximation the thermal density of $i$-th hadron sort is

$$
\varphi_{i}=\gamma_{s}^{S_{i}} g_{i} \int_{M_{i}}^{\infty} d m f\left(m, m_{i}, \Gamma_{i}\right) \int \frac{d \mathbf{k}}{(2 \pi)^{3}} e^{-\frac{\sqrt{m^{2}+\mathbf{k}^{2}}}{T}}
$$

Here $M_{i}$ is the dominant decay channel threshold and $f$ is the normalized Breit-Wigner mass attenuation. Thermal multiplicities $N_{i}^{t h}=V \frac{\partial p}{\partial \mu_{i}}$ ( $V$ is the effective emission volume) should be corrected by the hadron decays after the CFO according to the branching ratios $B r_{l \rightarrow i}$ which define the probability of particle $l$ to decay into a particle $i$. Hence the ratio of full multiplicities can be cast as

$$
R_{i j} \equiv \frac{N_{i}^{t o t}}{N_{j}^{t o t}}=\frac{p_{i}+\sum_{l \neq i} p_{l} B r_{l \rightarrow i}}{p_{j}+\sum_{l \neq j} p_{l} B r_{l \rightarrow j}} .
$$

This expression was used in our analysis of experimental hadron yields since it allows one to exclude $V$ and, hence, to reduce the number of fitting parameters. Here we fit the high quality experimental multiplicities measured at AGS for $\sqrt{s}_{N N}=2.7,3.3,3.8,4.3,4.9$ $\mathrm{GeV}\left[19\right.$ 27], the NA49 data measured at SPS energies $\sqrt{s}_{N N}=6.3,7.6,8.8,12.3,17.3 \mathrm{GeV}$ [28 36] and the STAR data measured at RHIC energies $\sqrt{s}_{N N}=9.2,62.4,130,200 \mathrm{GeV}$ [37]. These experimental data allow us to construct 111 independent ratios measured at 14 values of collision energies [4, 13]. However, from our past experience of successful fitting we learned that at any collision energy it is practically impossible to simultaneously reach the mean values of all independent hadron multiplicity ratios and hence the values of error bars are important. On the other hand, we would like to stress that some ratios have essentially smaller error bars than the other ones. Therefore, inclusion of such dependent ratios into a fit is important because it may affect some fitting parameters, since their errors are independent on the errors of other ratios. In particular, at AGS and SPS energies the ratio $\Lambda / p$ has rather small error bars (see the upper panel of Fig. 3). One should also remember that an exclusive role of $\Lambda$-hyperon was demonstrated recently in [14], where it was shown that the high quality simultaneous description of $K^{+} / \pi^{+}, \Lambda / \pi^{-}$and $\bar{\Lambda} / \pi^{-}$ratios requires introduction of an independent hard-core radius for $\Lambda$ hyperons. Therefore, to study the role of the $\Lambda / p$ ratio on the total fit quality we included it into our data sample and obtained 121 hadron multiplicity ratios to analyze.

The local fitting parameters for each collision energy $\left(T, \mu_{B}, \mu_{I 3}\right.$ and $\left.\gamma_{s}\right)$ and the global ones, i.e. the values of hard-core radii $R_{b}=0.385 \mathrm{fm}, R_{m}=0.41 \mathrm{fm}, R_{\pi}=0.19 \mathrm{fm}, R_{K}=$ 
$0.39 \mathrm{fm}$ and $R_{\Lambda}=0.09 \mathrm{fm}$, were determined by minimizing the quantity

$$
\chi^{2}=\sum_{p=1}^{121} \frac{\left(R_{p}^{\text {theor }}-R_{p}^{\text {exp }}\right)^{2}}{\sigma_{p}^{2}}
$$

where the summation is carried out over all data points. Here $\sigma_{p}$ is an experimental error of ratio $R_{p}^{e x p}$. The minimization of (4) showed that compared to previous results [4, 13, 14] the values of local fitting parameters $T, \mu_{B}, \mu_{I 3}$ and $\gamma_{s}$ are practically the same (i.e. within the errors bars they simply coincide), while the values of hard-core radii are changed. The present fit corresponds to a slight increase of $R_{b}, R_{m}$ and $R_{K}$. This makes their values practically the same, while $R_{\Lambda}$ demonstrate a slight decrease. The largest increase is experienced by the pion hard-core radius $R_{\pi}$, which almost doubles. The achieved fit quality $\chi^{2} /$ dof $=63.98 / 65 \simeq 0.98$ is about $3 \%$ larger than the one found in [14]. The results of $\Lambda / p$ fit are shown in the upper panel of Fig. 3. Compared to the $\Lambda / p$ ratio which was found in [3] (see Fig. 14 in [3]) without including it into a fitting procedure, the results of the upper panel of Fig. 3 demonstrate a sizable improvement of the $\Lambda / p$ ratio for $\sqrt{s_{N N}}=2.7-7.9$ $\mathrm{GeV}$, while at higher collision energies the description of this ratio slightly worsens. The quality of description of other ratios remains the same as in [14]. Also we checked that the sharp peak of the trace anomaly at CFO (see the upper panel of Fig. 1) is not affected by the change of pion hard-core radius. It turs out that inclusion of the $\Lambda / p$ ratio into a fit is important because it improves the description of this ration and, hence, it provides a higher confidence in our predictions for NICA and FAIR experiments which are related to the $\Lambda / p$ ratio.

Fig. 3 clearly shows that there are three regimes in the energy dependence of the $\Lambda / p$ ratio: at $\sqrt{s_{N N}}=4.3 \mathrm{GeV}$ the slope of this ratio clearly increases, while above $\sqrt{s_{N N}}=7.6$ $\mathrm{GeV}$ it nearly saturates. The change of slope at $\sqrt{s_{N N}}=4.3 \mathrm{GeV}$ is very similar to the prediction of Ref. [38] that the mixed phase formation can be identified by a rapid increase in the number of strange quarks per light quarks. This ratio is a convenient indicator because at low collision energies $\Lambda$ hyperons are generated in collisions of nucleons. As it is seen from Fig. 3, this mechanism works up to $\sqrt{s_{N N}}=4.3 \mathrm{GeV}$, while an appearance of the mixed phase should lead to an increase in the number of strange quarks and antiquarks due to the annihilation of light quark-antiquark and gluon pairs. Note that this simple picture is in line with the result that the mixed phase can be reached at $\sqrt{s_{N N}}=4.3 \mathrm{GeV}$, while QGP is formed at $\sqrt{s_{N N}} \geq 4.9 \mathrm{GeV}$. The dramatic change of the experimental $\Lambda / p$ ratio 
slope at $\sqrt{s_{N N}}>7.6 \mathrm{GeV}$ which is seen in Fig. 3 can be an evidence for the second phase transformation, which we discussed in the preceding section.

From Fig. 4 one can see even more dramatic changes in the collision energy dependence of two new ratios $\frac{\Delta \Lambda}{\Delta p}=\frac{\Lambda-\bar{\Lambda}}{p-\bar{p}}$ and $\frac{\Delta K^{+}}{\Delta p}=\frac{K^{+}-\bar{K}^{+}}{p-\bar{p}}$. Both quantities demonstrate a strong jump right in the collision energy region which is associated with the mixed phase formation, i.e. for $\sqrt{s_{N N}}=4.3-4.9 \mathrm{GeV}$. Also in Fig. 4 one can clearly see a change of the $\frac{\Delta \Lambda}{\Delta p}$ and $\frac{\Delta K^{+}}{\Delta p}$ slopes at $\sqrt{s_{N N}}=9.2 \mathrm{GeV}$. Our hypothesis is that the collision energy dependence of the $\frac{\Delta \Lambda}{\Delta p}$ and $\frac{\Delta K^{+}}{\Delta p}$ ratios is an indicator of two phase transformations. Since the observed jump of these ratios is located in the collision energy region of the mixed phase formation (i.e. a first order phase transition), then a change of their slope at $\sqrt{s_{N N}}=9.2 \mathrm{GeV}$ can be naturally associated with a weak first order or a second order phase transition. Note that such a hypothesis is well supported by the results of the meta-analysis [10] which we summarized above.

In contrast to other multiplicity ratios these ratios have a clear physical meaning of strange charge of $\Lambda$ hyperons (of $K^{+}$mesons) per baryon charge of protons. Also we would like to stress that at low collision energies the antibaryons are absent and, hence, $\frac{\Delta \Lambda}{\Delta p} \simeq \Lambda / p$. This can be easily seen from a comparison of Fig. 3 with the upper panel of Fig. 4. As one can see from the upper panel of Fig. 4 the behavior of thermal multiplicities of the $\frac{\Delta \Lambda}{\Delta p}$ ratio is very similar to the one of total multiplicities at $\mathrm{CFO}$ and, therefore, such a ratio maybe used to directly access the moment of CFO by other models which, in contrast to HRGM, do not account for the resonance decays.

\section{SUMMARY}

Here we briefly describe the peculiar irregularities at chemical freeze-out which, as we argue, can be used as the signals of the mixed phase/QGP formation. Two entirely different approaches presented here give the evidence that there is a possibility to experimentally detect two phase transitions in QCD. First of them is the first order phase transition to the quark-gluon-hadron mixed phase which, as we expect, can be formed at collision energies $\sqrt{s_{N N}}=4.3-4.9 \mathrm{GeV}$. The nature of the second phase transition is not fully understood now, but we are arguing that this can be either the weak first order or the second order

phase transition, which can be probed at collision energies $\sqrt{s_{N N}}=10-13.5 \mathrm{GeV}$. In the 
former case the initial states formed at the beginning of nuclear-nuclear collision leave QGP and return back to the mixed phase at these collision energies. In the latter case the initial states formed at the beginning of nuclear-nuclear collision cross the curve of the second order phase transition which maybe either the (partial) restoration of chiral symmetry or a transition between the quarkyonic matter and QGP [11]. Note that the irregularities/signals found at these collision energies can be studied at NICA and FAIR.

In this work we also present the results of an advanced fit of 121 hadron multiplicity ratios measured at AGS, SPS and RHIC. The HRGM with multicomponent repulsion allowed us to get a nearly perfect description of the $\Lambda / p$ ratio for the collision energies $\sqrt{s_{N N}}=2.7-7.9$ $\mathrm{GeV}$ and to keep the high quality of description of other hadronic multiplicity ratios with the total quality $\chi^{2} / d o f \simeq 0.98$. Such a high fit quality gives us a high confidence that the suggested ratios $\frac{\Lambda-\bar{\Lambda}}{p-\bar{p}}$ and $\frac{K^{+}-\bar{K}^{+}}{p-\bar{p}}$ can serve as safe indicators of discussed phase transitions. Hopefully, such ratios can be used at NICA and FAIR to locate the threshold energies of these transitions.

Acknowledgements. The authors are thankful to D. B. Blaschke, T. Galayuk, R.A. Lacey, I. N. Mishustin, D. H. Rischke, K. Redlich, L. M. Satarov and K. Urbanowski for discussions and valuable comments. K.A.B., V.V.S., A.I.I, D.R.O. and G.M.Z. acknowledge the partial support of the program "On perspective fundamental research in high-energy and nuclear physics" launched by the Section of Nuclear Physics of NAS of Ukraine.

[1] A. N. Sissakian, A. S. Sorin, M. K. Suleymanov, V. D. Toneev and G. M. Zinovjev, Phys. Part. Nucl. Lett. 5, (2008) 1.

[2] K. A. Bugaev et al., Phys. Part. Nucl. Lett. 12, (2015) 238.

[3] K. A. Bugaev et al., arXiv:1412.0718 [nucl-th] and references therein.

[4] K. A. Bugaev et al., Europhys. Lett. 104, (2013) 22002.

[5] A.V. Merdeev, L.M. Satarov and I.N. Mishustin, Phys. Rev. C 84, (2011) 014907.

[6] Sz. Borsanyi et al., JHEP 1208, (2012) 053.

[7] K. A. Bugaev, M. I. Gorenstein, B. Kämpfer and V. I. Zhdanov, Phys. Rev. D 40, (1989) 2903.

[8] K. A. Bugaev, M. I. Gorenstein and D. H. Rischke, JETP Lett. 52, (1990) 1121. 
[9] K. A. Bugaev, M. I. Gorenstein and D. H. Rischke, Phys. Lett. B 255, (1991) 18 and references therein.

[10] V. A. Kizka, V. S. Trubnikov, K. A. Bugaev and D. R. Oliinychenko, arXiv:1504.06483 [hepph].

[11] A. Andronic et al., Nucl. Phys. A 837, (2010) 65.

[12] D.R. Oliinychenko, K.A. Bugaev and A.S. Sorin, Ukr. J. Phys. 58, (2013) 211.

[13] K. A. Bugaev, D. R. Oliinychenko, A. S. Sorin, G. M. Zinovjev, Eur. Phys. J. A 49, (2013) 30.

[14] V. V. Sagun, Ukr. J Phys. 59, (2014) 755.

[15] V. V. Sagun et al., Ukr. J. Phys. 59, (2014) 1043.

[16] A. Andronic, P. Braun-Munzinger and J. Stachel, Nucl. Phys. A 772, (2006) 167 and references therein.

[17] J. Rafelski, Phys. Lett. B 62, (1991) 333.

[18] S. Wheaton, J. Cleymans and M. Hauer, Comput. Phys. Commun. 180, (2009) 84.

[19] J.L. Klay et al., Phys. Rev. C 68, (2003) 054905.

[20] L. Ahle et al., Phys. Lett. B 476, (2000) 1.

[21] L. Ahle et al., Phys. Lett. B 490, (2000) 53.

[22] C. Pinkenburg et al.,Nucl. Phys. A 698, (2002) 495.

[23] J. Barrette et al., Phys. Rev. C 62, (2000) 024901.

[24] L. Ahle et al., Phys. Rev. C 60, (1999) 064901.

[25] L. Ahle et al., Phys. Rev. C 58, (1998) 3523.

[26] S. Albergo et al., Phys. Rev. Lett. 88, (2002) 062301.

[27] B. B. Back et al., Phys. Rev. Lett. 87, (2001) 1970.

[28] I. G. Bearden et al., Phys. Rev. C 66, (2002) 044907.

[29] S. V. Afanasiev et al., Phys. Rev. C 66, (2002) 054902.

[30] S. V. Afanasiev et al., Phys. Rev. C 69, (2004) 024902.

[31] C. Alt et al., Phys. Lett.B 635, (2006) 270.

[32] T. Anticic et al., Phys. Rev. Lett. 93, (2004) 022302.

[33] S. V. Afanasiev et al., Phys. Lett. B 538, (2002) 275.

[34] C. Alt et al., Phys. Rev. Lett. 94, (2005) 192301.

[35] F. Antinori et al., Phys. Lett. B 595, (2004) 65. 
[36] F. Antinori et al.,J. Phys. G 31, (2005) 1345.

[37] J. Adams et al., Phys. Rev. Lett. 92, (2004) 112301.

[38] J. Rafelski and B. Müller, Phys. Rev. Lett. 48, (1982) 1066. 

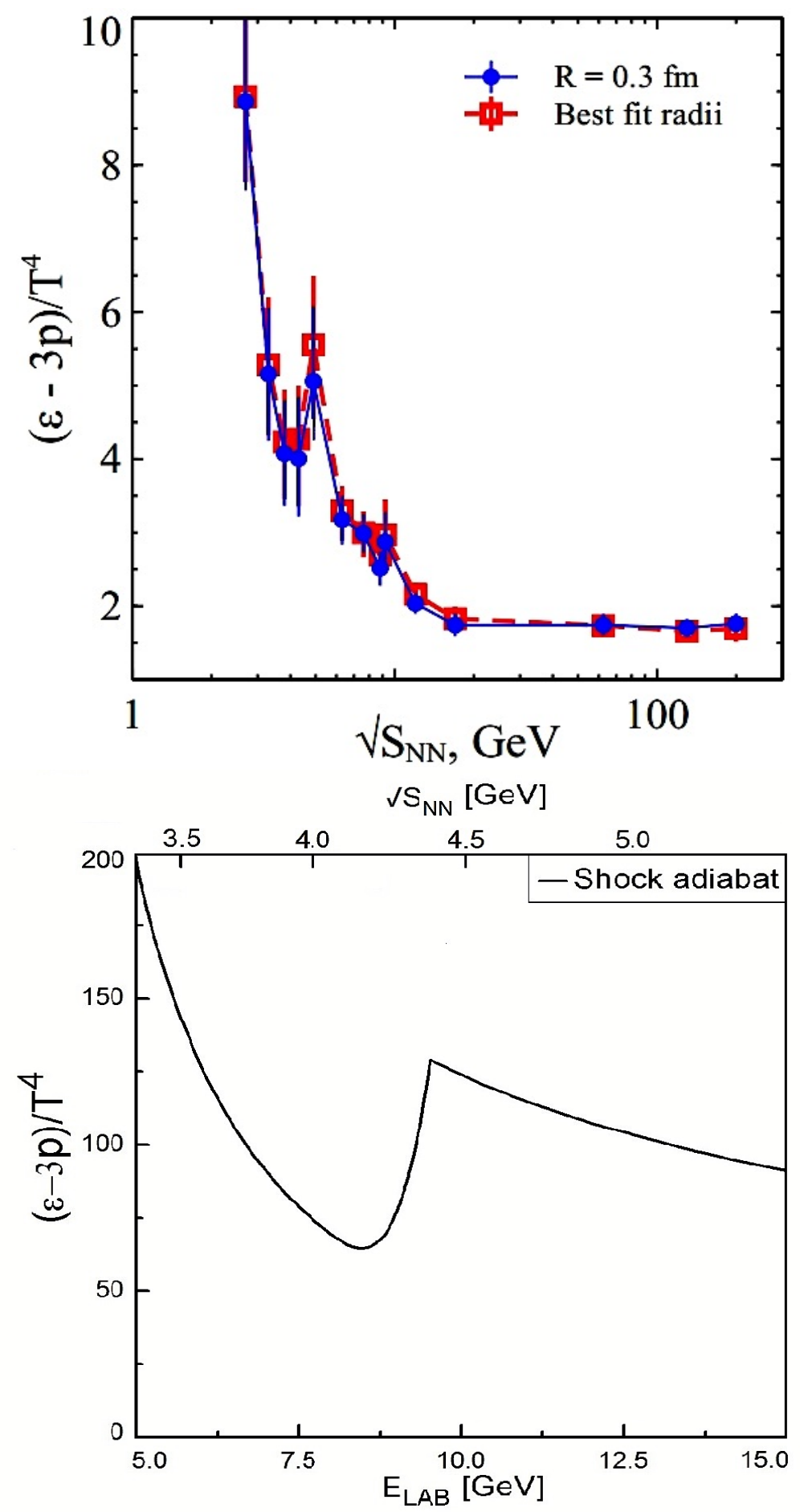

FIG. 1: Trace anomaly as function of collision energy at CFO (upper panel) [3] and along the shock adiabat [2] (lower panel). The details on the set of best fit hard-core radii shown here are given in [4]. 


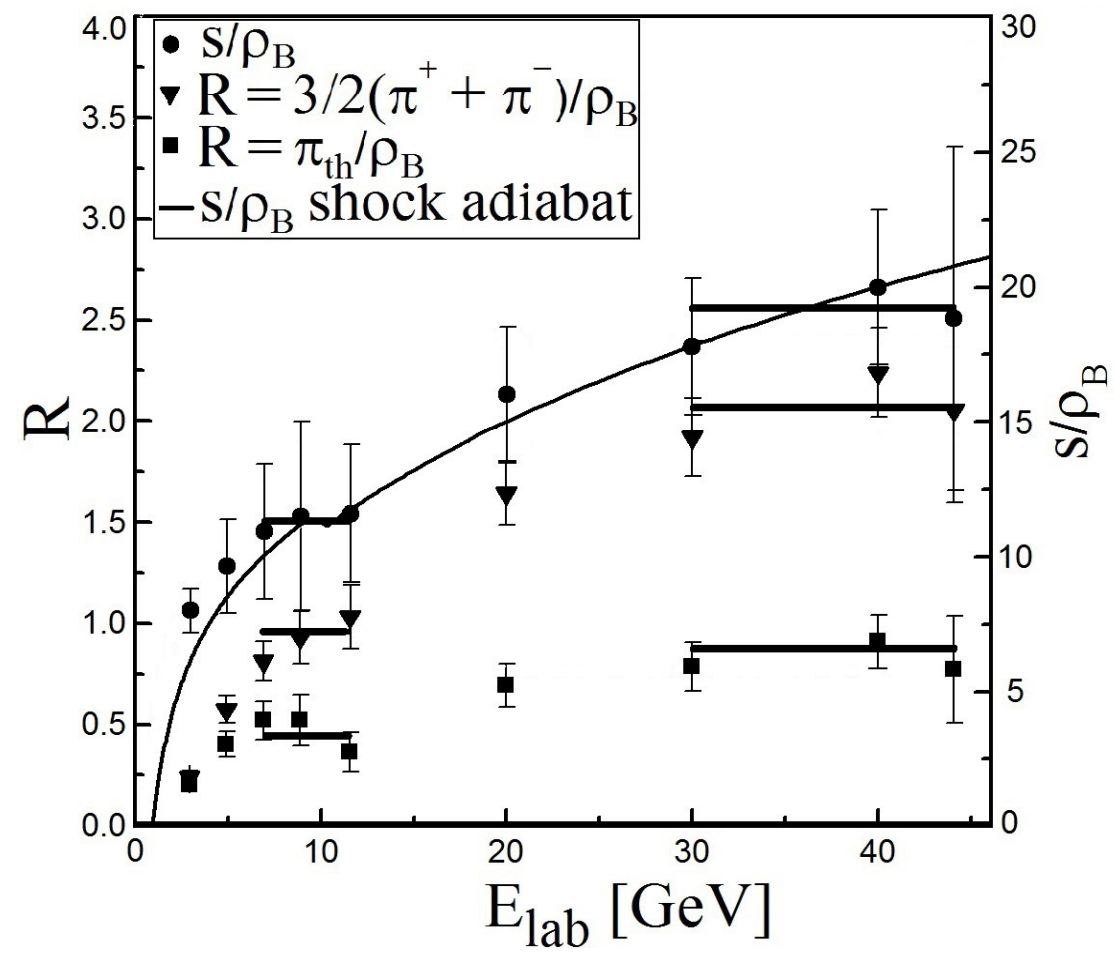

FIG. 2: The correlated quasi-plateas at CFO are taken from [2] (see details in the text).

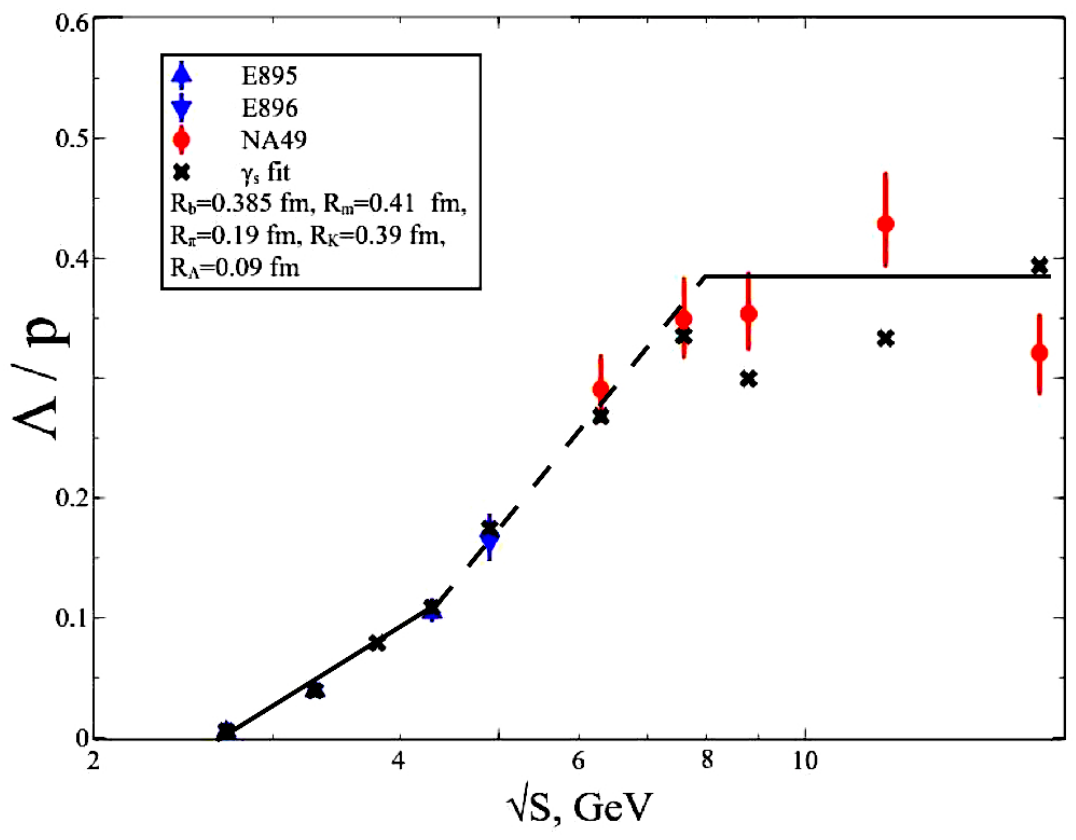

FIG. 3: The collision energy dependence of the $\Lambda / p$ ratio obtained within the present HRGM. The lines are given to guide the eye. More explanations tare given in the text. 

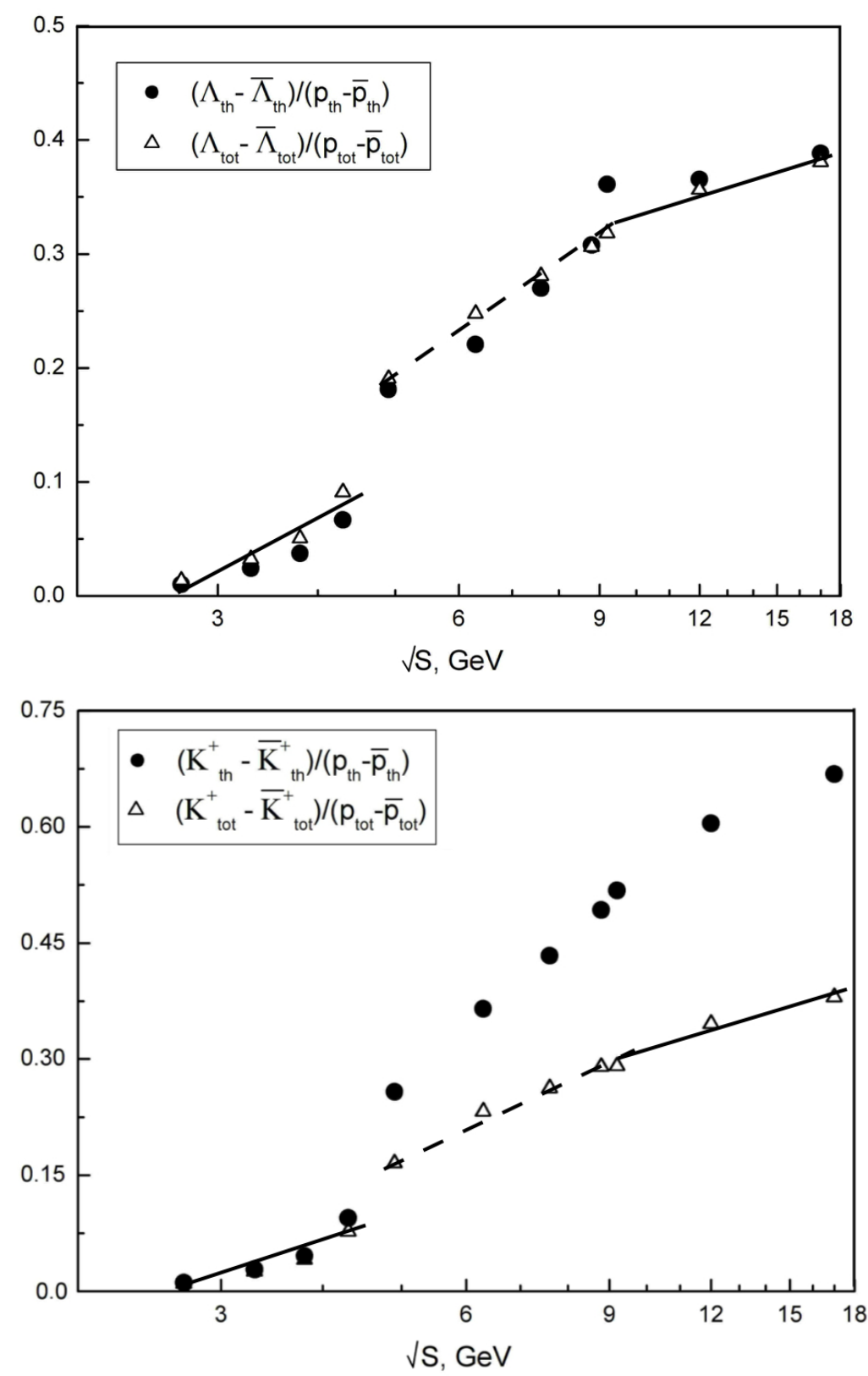

FIG. 4: Predictions for the collision energy dependence of $\frac{\Delta \Lambda}{\Delta p}$ (upper panel) and $\frac{\Delta K^{+}}{\Delta p}$ (lower panel) ratios. The triangles depict the total multiplicities, while the circles correspond to the thermal multiplicities. The lines are given to guide the eye. 